\section{SHORT TRACK VS HOCKEY HELMETS: USING FINITE ELEMENT ANALYSIS TO COMPARE STRAIN TO THE BRAIN}

${ }^{1}$ Daniel Aponte, ${ }^{2}$ Suzanne Lederc, ${ }^{1}$ David Pearsall. 'McGill University, Montreal, Canada; ${ }^{2}$ Institut National du Sport du Québec, Montreal, Canada

\subsection{6/bjsports-2021-IOC.393}

Background Finite element analysis (FEA) is a computational modeling method widely used in materials and mechanical engineering to simulate the strain in a given physical system. The SIMon (Simulation Injury Monitor) is a finite element head model developed by the National Highway Traffic Safety Association in order to study how various impact conditions affect the human brain.

Objective Compare brain strain in high and low velocity impacts, between short track (ST) and ice hockey (IH) helmets.

Design Two-group experimental design.

Setting Data from previous impacts used in SIMon to model the human brain response to impacts.

Patients (or Participants) 5 different helmet models; 3 ST models and $2 \mathrm{IH}$ models.

Interventions (or Assessment of Risk Factors) Assessment of ST and $\mathrm{IH}$ helmet impact attenuation under various conditions.

Main Outcome Measurements Cumulative Strain Damage Measure (CSDM) 15, 20 and 25. CSDM is the percentage of brain volume that crosses the 15\%,20\% and 25\% threshold. This has been shown to correlate with deformation-related brain injuries, such as Diffuse Axonal Injury.

Results One-way between-helmet ANOVAs for CSDM 15, 20 and 25 in low and high velocity impacts revealed statistical differences in CSDM 15, 20 and 25 [CSDM 15, F(4, 34) = 70.7, p<0.05; CSDM 20, F(4, 34) = 63.4, p<0.05; CSDM $25, \mathrm{~F}(4,34)=32.5, \mathrm{p}<0.05]$. The trend was that ST helmets outperformed $\mathrm{IH}$ helmets in rear, rear-boss and front-boss impacts, but that IH helmets outperformed ST in side impacts.

Conclusions The results of the FEA reveal a difference between the ST and IH helmets, with ST helmets generally outperforming $\mathrm{IH}$ helmets. Interestingly, these results are different than the results optained when comparing linear and rotational acceleration results for these same impacts. Currently, certifications only require peak linear acceleration values be below a certain threshold. However, these studies demonstrate the importance of using various outcome measures to determine the efficacy of helmets in sport.

\section{A NOVEL VIRTUAL HELMET FIT ASSESSMENT FOR ICE HOCKEY AND RINGETTE PLAYERS AMIDST THE COVID- 19 PANDEMIC}

\footnotetext{
1,2,3 Ash T Kolstad, 1,2 Linden C Penner, ${ }^{1,2}$ Alexandra J Sobry, 1,2,3,4,5Amanda M Black, 1,2,3,4,5,6,7 Brent E Hagel, 1,2,3,4,5,6,7,8 Carolyn A Emery. ${ }^{1}$ Sport Injury Prevention Research Centre, Faculty of Kinesiology, University of Calgary, Calgary, Canada; 'Integrated Concussion Research Program, University of Calgary, Calgary, Canada; ${ }^{3}$ Alberta Children's Hospital Research Institute, University of Calgary, Calgary, Canada; ${ }^{4}$ Hotchkiss Brain Institute, University of Calgary, Calgary, Calgary, Canada; ${ }^{5} \mathrm{O}^{\prime}$ Brien Institute for Public Health, University of Calgary, Calgary, Canada; ${ }^{6}$ Department of Paediatrics, Cumming School of Medicine, University of Calgary, Calgary, Canada; 'Department of Community Health Sciences, Cumming School of Medicine, University of Calgary, Calgary, Canada; ${ }^{8} \mathrm{McC}$ aig Institute for Bone and Joint Health, University of Calgary, Calgary, Canada
}

Background Proper helmet fit is an important consideration for preventing head injuries, including concussions, in helmeted sports like youth ice hockey and ringette. Helmet fit assessments are typically completed in-person; however, this was not possible given COVID-19 restrictions. Thus, alternative considerations for virtual assessments were required.

Objective To examine the feasibility and inter-rater reliability of virtual ice hockey and ringette helmet fit assessments.

Design Cross-sectional.

Setting Calgary, Canada.

Participants Elite/upper division youth (ages 13-18) ice hockey $(\mathrm{n}=31$ males) and ringette $(\mathrm{n}=30$ females) players.

Assessment of Risk Factors Standardized ice hockey/ringette helmet fit criteria were developed and reliable for in-person assessments. Criteria were adapted for virtual delivery to participants over ZOOM video platform individually by two trained assessors per sport.

Main Outcome Measurements Twelve helmet fit criteria scored as yes/proper fit or no/poor fit were used to assess helmet shell fit (e.g., helmet fits snug, doesn't cover eyes), positioning (e.g., helmet is 1-2 finger widths above eyebrows, covers base of skull), facemask fit (e.g., chin piece fits, facemask does not move left/right), and others. Percent agreement (PA) between raters was used to describe inter-rater reliability, and each rater documented barriers for completing the assessments virtually.

Results Acceptable PA (>80\%) was demonstrated for $8 / 12$ criterion for ice hockey and 9/12 for ringette. Below acceptable agreement was found for all four criterion assessing the helmet facemask fit (PA range: 48\%-74\%) in ice hockey players and criteria for the chin straps fit $(\mathrm{PA}=66 \%)$, helmet positioning $(\mathrm{PA}=73 \%)$, and facemask fit $(\mathrm{PA}=63 \%)$ in ringette players. Common barriers were related to technology (e.g., audio/video quality) and environment (e.g., noisy, lighting).

Conclusions Virtual helmet fit assessments are feasible and reliable for most criteria, with more training required for criteria below acceptable agreement. Virtual assessments provides another option for assessing helmet fit for concussion prevention in helmeted sports.

\section{PROTECTIVE EQUIPMENT IN YOUTH ICE HOCKEY: ARE MOUTHGUARDS AND HELMET AGE RELEVANT IN EVALUATING CONCUSSION RISK?}

\footnotetext{
1,2,3 Ash T Kolstad, 1,2,3,4 Paul H Eliason, ${ }^{1,2}$ Jean-Michel Galarneau, 1,2,3,4,5 Amanda M Black, 1,2,3,4,5,6,7 Brent E Hagel, 1,2,3,4,5,6,7,8 Carolyn A Emery. ${ }^{1}$ Sport Injury Prevention Research Centre, Faculty of Kinesiology, University of Calgary, Calgary, Canada; 'Integrated Concussion Research Program, University of Calgary, Calgary, Canada; ${ }^{3}$ Alberta Children's Hospital Research Institute, University of Calgary, Calgary, Canada; ${ }^{4}$ Hotchkiss Brain Institute, University of Calgary, Calgary, Canada; ${ }^{5} \mathrm{O}^{\prime}$ Brien Institute for Public Health, University of Calgary, Calgary, Canada; ${ }^{6}$ Departments of Pediatrics, Cumming School of Medicine, University of Calgary, Calgary, Canada; ${ }^{7}$ Community Health Sciences, Cumming School of Medicine, University of Calgary, Calgary, Canada; ${ }^{8} \mathrm{McC}$ aig Institute for Bone and Joint Health, University of Calgary, Calgary, Canada
}

\subsection{6/bjsports-2021-IOC.395}

Background The high concussion burden in youth ice hockey is concerning. An important yet understudied area for prevention is protective equipment (e.g., wearing a mouthguard, age of helmet).

Objective To compare rates of concussion between players based on mouthguard use and helmet age. 